\title{
Wellbeing as a proxy for a mHealth study
}

\author{
Chonlatee Khorakhun \\ University of St Andrews, UK \\ ck46@st-andrews.ac.uk
}

\author{
Saleem N. Bhatti \\ University of St Andrews, UK \\ saleem@st-andrews.ac.uk
}

\begin{abstract}
The quantified-self is a key enabler for mHealth. We propose that a wellbeing remote monitoring scenario can act as a suitable proxy for mHealth monitoring by the use of an online social network (OSN). We justify our position by discussing the parallelism in the scenario between purpose-driven wellbeing and mHealth scenarios. The similarity between these two scenarios in terms of privacy and data sharing is discussed. By using such a proxy, some of the legal and ethical complexity can be removed from experimentation on new technologies and systems for mHealth. This enables technology researchers to carry out investigation and focus on testing new technologies, system interactions as well as security and privacy in healthcare in pre- clinical experiments, without loss of context. The analogy between two purpose-driven scenarios, i.e. fitness monitoring in wellbeing scenario and remote monitoring in mHealth, is discussed in terms of a practical example: we present a prototype using a wellbeing device - Fitbit - and an open source online social media platform (OSMP) - Diaspora.
\end{abstract}

\section{INTRODUCTION}

With an increasing number of citizens worldwide, including an ageing population [1], a higher burden in healthcare [2] drives a shift of global concerns from dealing with acute illnesses, to management of chronic illnesses. A key part of such a move is prevention and early detection through routine monitoring for diagnosis. However, monitoring a growing population is challenging for limited healthcare resources.

mHealth enables the use of mobile devices with ubiquitous communication for pervasive monitoring for the collection of bio-data outside clinical environments. This could reduce healthcare costs, improve patients care due to pervasive monitoring, as well as improve patients quality of life. We have proposed the use of an online social media platform (OSMP) as a platform to enable pervasive and seamless health monitoring [3]-[6]. By exploiting existing, publicly accessible infrastructure, initial costs can be reduced, as well as allowing fast and flexible application development at scale. An OSMP presents familiar interfaces for patients and healthcare professionals, and also allows application developers to work with a set of technologies that are widely used and well-known.

\section{A. Change in stakeholder focus}

The traditional healthcare delivery models are changing towards a patient-driven focus, with a shift of control from doctors to patients. The collection of patient-generated biodata is an essential part of an mHeatlh monitoring system, aided by the maturity of technology and systems, with availability of ubiquitous communication, self-tracking devices, and self-monitoring for maintaining the quantified self [7].
In a conventional healthcare model, almost all measurements are performed at clinical sites (such as hospitals and clinics) and under the supervision of professional healthcarers, while patients have no access to their own data. In a patientdriven healthcare model, patients can measure their own health data and share it as they see fit. Due to the freedom and control enabled by self-monitoring and self-tracking, it is therefore prudent to investigate the privacy and data sharing in this new environment of the quantified self and its application to a healthcare model, i.e. with whom and to which extent people are willing to share their private health data.

\section{B. New privacy and security concerns}

Previous studies on privacy and data sharing in healthcare have been based largely on interviews and focus on sharing of heatlh data in general, i.e. what are privacy concerns in sharing health data in public, with friends, family and third parties? In this work, we are interested in studying the sharing behaviours of people in a closed and secure environment, i.e. privacy concern and sharing of sensitive data with the trusted parties of a closed healthcare environment.

In order to conduct an experiment for research in healthcare, a key challenge for investigators is to use sensitive health data. In previous studies, this has involved cooperation with professional medical staff as well as real patients who are volunteers. This creates many ethical, medical and pastoral concerns that are very complex. For example, what would happen if during the experiment, a software developer during software testing discovers that a volunteer has a serious illness that was previously undiagnosed?

\section{Proxy studies: the quantified self}

Many studies being conducted at the moment are related to investigating technology and systems and so the challenge is how to conduct those experiments and development, in a pre-clinical setting, without losing context and relevance to he eventual clinical application.

Therefore, in this work, we propose the use of a wellbeing experiment as a proxy for mHealth. Or experiment is to enable the study of privacy and data sharing, and we will show that there is an analogy and equivalence between these two scenarios, i.e. there exists a similarity in both of these purposedriven scenarios. We take a position that since our aim is to investigate the technology, system interaction and privacy of data sharing strong analogies can be made with a mHealth scenario, but without the risk of using real health data. A key 
aspect of our approach is the model of interaction between the stakeholders and actors in each scenario, for which we exploit existing social relationships.

\section{Structure of this paper}

After covering related work in Section II, we describe our remote monitoring application (RMA) and model of a carer network in Section III. The roles equivalence model and design description of the prototype experiment - using a Fitbit device and the Diaspora platform - is presented in Sections IV and $\mathrm{V}$ respectively. A discussion with respect to privacy and data sharing, including comparison between our prototype and original Fitbit application, is given in Section VI. Finally, we summarise and conclude our discussion in Section VII.

\section{RELATED WORK}

\section{A. Quantified self for mHealth}

Based on available software development kits (SDKs) for popular mobile operating systems and present technologies, there are a number of smartphone applications and devices for quantified self-tracking available. These range from general wellbeing applications (monitoring fitness and nutrition) (e.g. FitBit [8], Jawbone UP [9], Basis [10], Nike+ [11], Adidas miCoach [12], Shine [13], Lark [14], Garmin [15], Philips Directlife [16], DailyMile [17], RunKeeper [18] and LoseIt [19]) to systems aiding diagnosis and treatment (e.g. AgaMatrix [20]). These applications enable users to update their health status and health goals via online portals accessed as a web service. To encourage personal health monitoring and health coaching, such applications can also connect users to existing online social networks to update and share their health data with friends and family.

The quantified-self is also considered as an essential part of mHealth with remote health monitoring applications which collect health data for healthcare professionals and/or for a Personal Health Record (PHR). The smartphone will is used as a monitoring platform to access health data or as an interface to users (both patients and doctors), i.e. as a portal to access health information [21] or to monitor users' own health-related behaviour [22].

Fitbit has almost $70 \%$ of market share ${ }^{1}$ as well as has been used in other research studies [23] [24] [25] [26]. Its advantages include accessible public APIs and a webbased portal. Experiments in [27] show a better accuracy of Fitbit compared to other wearable devices recording physical activity. Fitbit has been used in a clinic setting to monitor the recovery of discharged patients at home [28] i.e. post-acutecare facilities. This is evidence that well-being monitors like Fitbit can be applied to remote health monitoring.

\section{B. Healthcare and social networking}

There has been an increasing use of social networking in healthcare such as Patientslikeme [29], Doximity [30],

\footnotetext{
${ }^{1}$ Fitbits Market Share Remains Strong Despite Force Recalls, Press Release, 23 Apr 2014, http://www.ubergizmo.com/2014/04/fitbits-market-shareremains-strong-despite-force-recalls/
}

Sermo [31] and HelloHealth [32]. These technologies refocus the patient-physician relationship [33] to patient-centric while enabling better collaboration and communication between patients, caregivers and physicians as well as improving healthcare efficiency.

In addition, social networking and online communities can help provide motivation. As a result, many existing health applications try to imitate the concept of an online social network by creating a community feeling, i.e. sharing and competing with friends and family, having friends or groups and having forums for social communication. An example is a fitness application developed in [34] which has socialisation features to compete and collaborate with friends. Also, mobile application like [24] builds a health social network to send and receive health tips and encouragement as well as acting as an interface to accumulate health and fitness activities from various fitness device, e.g. Fitbit, into one interface. There has also been an increased interest in closed-loop healthcare which enables patients to receive feedback to improve their heatlhcare, e.g. applications in [35] and [36] provide feedback of users' current wellbeing states to promote awareness and improve health levels.

We have proposed the concept of a carer network and the use of an OSMP [3]-[6] to integrate both closed loop and social network functionalities for future mHealth. Based on a social interaction in an OSMP, collaboration and communication in a carer network can be enabled. Due to sensitivity of health data, we have proposed in [5] that it is possible to achieve security and privacy of private health data as well as considerable functionality for mHealth monitoring by the use of a completely open source OSMP.

\section{Privacy and information sharing}

The sensitivity of health data coupled with the relative freedom of sharing data for the quantified-self raises concerns for privacy, e.g. what type of health-related data should be shared, with whom and in which situation? There are a range of studies in this area, e.g. a study in [37] investigates sharing of health data in online community, whereas the study in [38] investigates fine-grained data sharing and considers social sharing for social support. So far, most investigations in this research area are based on surveys and user interviews. To the best of our knowledge, an experiment by Prasad et al [25] is the first study that collects data and investigates privacy concern related to data sharing with real user interactions. Such studies are resource intensive, difficult to execute and reproduce, and can take a considerable amount of time.

Similar to our experiment, the study by Prasad et al [25] investigates information sharing behaviour in mHealth, when users have options to share with friends, family, third parties and the public. However, we are interested in investigating the sharing behaviour in a purpose-driven mHealth scenario, i.e. sharing in purpose-oriented and trusted environment of a carer network, i.e. non public. Despite the concern in sharing of private health data, the interview in [39] shows that people are willing to share their sensitive health data if the data is 
seen as useful in helping to provide care. However, patients' decisions could change from time to time (for example, if a new diagnosis based on collected data is found) and therefore any experiments with real clinical data run the risk of exposing sensitive data to non-clinical staff.

\section{Experiments and trials for mHealth}

mHealth trials which collect sensitive health data require prfoessional medical staff and clinical approval. Of course, before systems are commissioned for use, such trials are required. Examples of trials on mHealth monitoring which deploy research prototypes, conduct in sensitive and high risk setting and require clinicians involved are [40], [41], [42] and [43]. However, at early stages, such clinical involvement is risky for clinical reasons and cumbersome for researchers. However, as wellbeing information is already shared by many users, and has a strong contextual link to a medical scenario, with suitable interaction models for users, it has the potential to act as an excellent proxy for mHealth monitoring.

\section{MHEALTH MONITORING}

We present a mHealth scenario in which we consider the use of an OSMP to enable two primitive functions: remote health monitoring and asynchronous alerts, in the context of a carer network. The analogy between the presented mHealth scenario in this section and the parallel well-being scenario will be discussed later in the next section.

\section{A. RMA using OSMP}

In our previous work, we have assessed the utility of OSMPs for implementing two primitive functions [6]: remote monitoring of personal bio-data [3] and generation of asynchronous alerts [4], as well as examined the use of a completely open source OSMP, Diaspora ${ }^{2}$ to provide better control over security and privacy as the OSMP can be run by the healthcare provider [5].

Figure 1 shows the mHealth remote monitoring scenario we consider and have previously examined. Personal bio-data is collected from a patient, sent to a server and may need to be accessed by several actors who are remote. The dashed (red) outline indicates our use of an OSMP as a portal to access to the collected health data within our scenario.

We focus on leveraging the existing infrastructure to reduce cost and enable pervasive monitoring, i.e. the use of an OSMP and open software for a remote monitoring application and Internet connectivity for network infrastructure. Employing existing infrastructure which is public, open, easily accessible to users and developers, has low costs to enter the market, and is subject to existing web standards, could yield great benefits. An open platform with open APIs and SDKs would also enable interoperability and extensibility, i.e. the application could work with a wide range of devices and medical systems. Previous mHealth and remote monitoring applications are custom-build, expensive and have taken a long time to develop.

${ }^{2}$ https://diasporafoundation.org
Using open source software based on web technologies would allow the application to be accessible on multiple platforms.

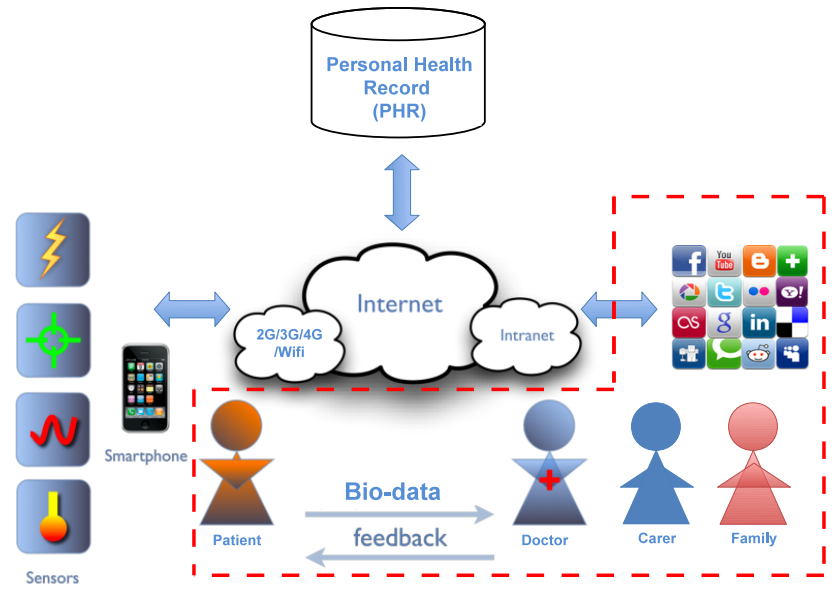

Fig. 1: A remote monitoring application (RMA) using an OSMP to form a carer-network. OSMP enables communication and colloboration in a carer-network as well as provides a portal to access the collected bio-data and to generage a message alert for an emergency situation.

\section{B. Carer network}

As can be seen from Figure 1, our remote monitoring application provides a portal to access the collected bio-data and provide the appropriate visibility and viewpoints for actors in a carer network. We have proposed the new concept of carer network which is based on the relationships in an existing healthcare regime that is common worldwide [44]. The carer network, works in harmony with, and provides support for, existing relationships and interactions between patients and healthcare professionals:

1) the doctor or consultant in charge of the management of the healthcare regime;

2) the professional carer who is a local contact for the patient and implements the clinical care (e.g. General Practitioner in the UK);

3) a family member or friend who is concerned about the patient and acts as informal caregivers (e.g. a neighbour for an elderly patient);

4) and the patient.

\section{The quantified self for mHealth}

In the context of mHealth, the mHealth application and actors in the carer network of Figure 1 exist within the the dashed (red) line. Outside that boundary, the systems and mechanisms are, effectively, for the purposes of measurement, monitoring and feedback. The core interactions and sharing of data (and therefore privacy concerns) are within the boundary. Outside the boundary, we are still concerned with security and privacy, but in the context of unauthorised access. This is out of scope for our study, but would be an important aspect of real system deployment.

The use of wellbeing data, for example the Fitbit device and portal, is outside the dashed (red) boundary. Fitbit provides 
measurement and monitoring, and, for our prototype (Section V), a mechanism for collection of bio-data. This can, of course, be replaced by another suitable system. Also, whilst we have said that concerns of unauthorised access are outside the scope of our current study, the danger of such compromise does exist within our use of Fitbit $^{3}$.

\section{WELlBEING MONITORING AS A PROXY}

In order to avoid risks of getting exposing with sensitive health data as well as removing the complexity and overhead of clinical studies, we design a wellbeing experiment which is a parallel scenario to mHelath monitoring, and can be used as a proxy for a mHealth scenario. In this section, we will justify the similarity between the two scenarios.

\section{A. Parallel scenario}

We consider a scenario of fitness monitoring as a parallel scenario for remote health monitoring. The two scenarios have similarity in terms of measurement of bio-data, people may use wearable sensors which collect information about physical condition and physical activities, e.g. steps, calories, sleep patterns, heart rate etc. These wellbeing data are collected and sent to a server for self- monitoring. Users can make decisions on how to share the data, e.g. with friends or family as well as analysis by a fitness and/or health professional to improve their fitness and health levels. Based on existing roles in a fitness and sport environment [45], we define a fitness network which is consists of four actors as follows:

1) A client is parallel role to the patient in the carer network.

The client is a person who wants to improve their health and fitness with a responsibility to follow an exercise plan, and try to achieve agreed goals.

2) Personal trainer, parallel role to the doctor in the carer network.

The trainer could be a service provided by staff from a gym, or could be a private, personal trainer. It is common that a trainer meets with clients for one or two sessions to clarify goals and design an exercise programme. The workout plan is created specifically subject to the clients need and physical abilities. The trainer helps clients to work toward their goals by setting weekly fitness goal and workout plan, motivating and providing feedback to clients as well as making sure that system is running and weekly goal is achieved. The exercise programme is very similar to a healthcare regime that would be established by e doctor or consultant.

3) Fitness coach, parallel role to the carer in carer network. The coach is responsible for the day-to-day monitoring of the adherence to the trainers's programme, and may provide motivational and corrective instructions. The coach's role is to implement the plan and motivate clients to work toward the fitness goal through daily

\footnotetext{
${ }^{3}$ Love your Fitbit? Be warned: Companies sell your data http://www.advisory.com/daily-briefing/2014/04/23/love-your-fitbit-bewarned-companies-sell-your-data
}

feedback and plan. This is similar to the role of the carer, who might be a local healthcare professional at a clinic, or local physician.

4) Fitness buddy, parallel role to the em family member in carer network

The buddy helps the clients to achieve their goal by motivating and/or working together. This could be a friend or family member, much as in the carer network.

We see a clear correspondence between these two purposeddriven scenarios of fitness network and carer network. There is correspondence between roles and a similarity in data sharing behaviour. Both scenarios are subject to purpose-oriented sharing, i.e. data is shared as expertise and permissions deem appropriate, and not for general access by thrid parties. For example, patients are willing to provide their real medical information to professional health providers since the data is useful to improve their healthcare. Similarly, clients are willing to share their physical activities with professional trainers to improve their fitness. This is because the sharing of information in both situations is based on trust, i.e. in order to achieve their goals (improve either fitness or health level), it is required to share the useful information.

In particular, if the sharing of data is appropriate to the role, then trust would exist. For example, clients would provide their steps information to their buddy, but might give away information about their heart-rate, even though they might give that information to the trainer or coach. Likewise, in the carernetwork, patients may not need to provide their heart-rate to family members, but would give it to the doctor.

Also, the sharing of fitness data here is more appropriate and analogous to analyses of sharing and privacy behaviour than, say, considering online privacy habits of people with respect to financial data or information about their hobbies or pastimes.

Of course, there is not an exact match of data sharing and concerns for privacy between the mHealth and wellbeing scenario. For example, there may be national data protection and privacy laws that govern use of personal health data. However, the differences would need to be evaluated and modified at a further level of study: our intention is to allow a more favourable environment for initial investigations an experimentation without the clinical overhead.

\section{A PROTOTYPE WELlBEING MHEALTH PROXY}

We describe the design of a prototype system using the Disapora ${ }^{4}$ open source OSMP and Fitbit ${ }^{5}$ activity trackers. We explain how the relationships modelled in the system relate to a mHealth scenario.

\section{A. Application outline}

Figure 2 shows our implementation, consisting of two parts: a measurement system and remote monitoring application $(R M A)$. In previous investigation [5], we have proposed the use of an open source OSMP - Diaspora - as a platform to

\footnotetext{
${ }^{4}$ https://diasporafoundation.org

${ }^{5}$ http://www.fitbit.com/
} 
enable the personal health monitoring while enabling flexible application development and modifications, allowing finegrained control of security \& privacy as well as reducing cost and allowing scalability of systems. In our application development, the Diaspora platform is modified to provide the RMA functions, as well as the interaction between the actors (patient, family, carer and doctor) in the carer networks of mHealth scenario

\section{B. Integrating the quantified self using Fitbit}

The analogy from the carer network to the fitness network (trainer, coach, buddy and client) in the fitness networks of the wellbeing scenario, can be realised via an online social network. We choose to investigate our implemented OSMP platform using Fitbit activity tracker devices [8] as a measurement system in our proxy wellbeing scenario, but a real medical application would use a different measurement system (e.g. wearable sensors collecting health bio-data in mHealth scenario). Diaspora uses the Fitbit API to access the data from the Fitbit server and store the data into the server. Each actor accesses the platform and will see a different viewpoint of monitored data depending on their roles in the fitness/carer network. It should be noted that, in an mHealth scenario, the viewpoint is controlled by an access control system configured by the healthcare provider, with appropriate consideration of patients personal privacy preferences, national laws, etc.

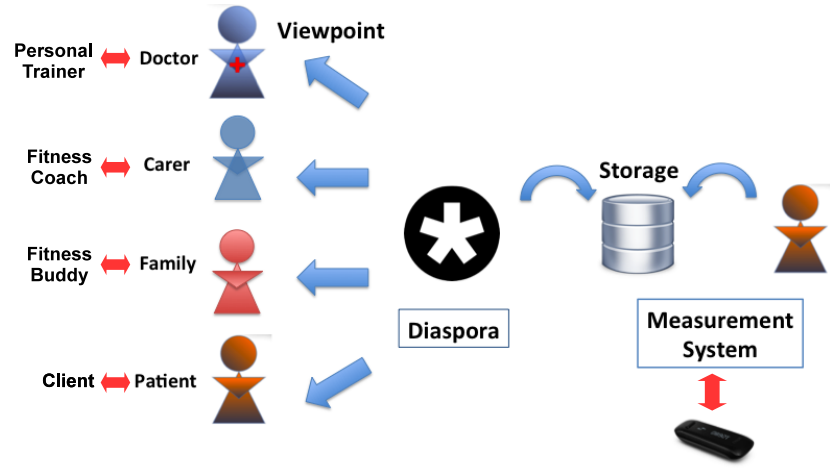

Fig. 2: A design of a wellbeing experiment used as a proxy for mHealth monitoring using online social network (OSN). Fitbit is used as a measurement system. An open source online social media platform (OSMP) - Diaspora - provides access to the stored Fitbit data for different actor viewpoints. The conventional actors in carer network (patient, family, carer and doctor) is replaced with parallel roles (client, fitness buddy, fitness coach and personal trainer) in wellbeing scenario.

The measurement system consists of sensors and systems to monitor and store personal bio-data. We use Fitbit as a measurement system just for convenience for our wellbeing scenario, but the design is modular allowing other measurements systems to be used for a real mHealth scenario. Fitbit is a commercial activity tracker, consisting of a wearable device and a web portal. The device measures data such as the number of steps taken daily, estimates of the calories burned and distances travelled. All data is synchronised automatically via Bluetooth to smartphones or via dongles to PCs, and uploaded to Fitbit web servers.

\section{Remote monitoring for mHealth}

The RMA is implemented in Diaspora which is a standardsbased web platform. Being a privacy-aware and decentralized OSMP, Diaspora servers allow users to stay in control of their data and are administered by individual users or organisations. Since Diaspora is an open source OSMP toolkit, it is possible that healthcare providers maintain their own Diaspora servers in order to keep complete control of security and privacy policies. In analogy to a healthcare provider server, we host a Diaspora using a local virtual machine server, with the Diaspora code extended to implement the RMA, access control (privacy) policies and to provide access to the Fitbit servers to collect measurement data.

Figure $3 \mathrm{a}, 3 \mathrm{~b}$ and $3 \mathrm{c}$ show application snapshots for doctor (trainer), carer (coach) and patient (client) viewpoints, allowing views of various Fitbit data in different visualisations, such as tables, meters and charts. The chart and meter graphics are from Google Charts to demonstrate the use of a mash-up for fast application development. To avoid privacy concerns, with bio-data, in a real mHealth application, such visualisations would be provided by the healthcare provider or a trusted party and not by Google Charts. In this example, the carer (coach), the family (buddy) and the patient (client) can access only the monitored Fitbit data for that day, whereas the doctor (trainer) has more detailed access to all historical Fitbit data also. The pie charts are used for analysis of daily Fitbit data which can be accessed only by the doctor (trainer) and the carer (coach). Another graphic of Fitbit data, in the form of a meter, is used to help the family member (buddy) and the patient (client) for quick data interpretation to achieve their exercise goals. Note that the patient (client) has the same viewpoint as the family member (buddy) in this case, and neither have access to the detailed data. All views are controlled by policy, so are configurable as required: we present a simple example to show the concept. Each actor has access only to the part of the data they require subject to their roles in a carer (fitness) network. Our Diaspora application is used as a portal to authorise each actor and provide an appropriate viewpoint and visualisations.

\section{DISCUSSION}

\section{A. Privacy and data sharing}

Our purpose for using a proxy experiment is to investigate the privacy and information sharing in mHealth monitoring scenario using the wellbeing monitoring scenario. We are interested in gaining insights of people's sharing behaviour in the trusted environment of a carer network. We will show that privacy concerns and data sharing in both parallel scenarios are similar.

In our designed experiment, clients' wellbeing data, i.e. steps and sleep patterns, will be collected by Fitbit and will be accessed by actors in a fitness network. Based on Diaspora interfaces, clients will have simple control over sharing of thier own data, i.e. simple turn on/off mechanisms. 

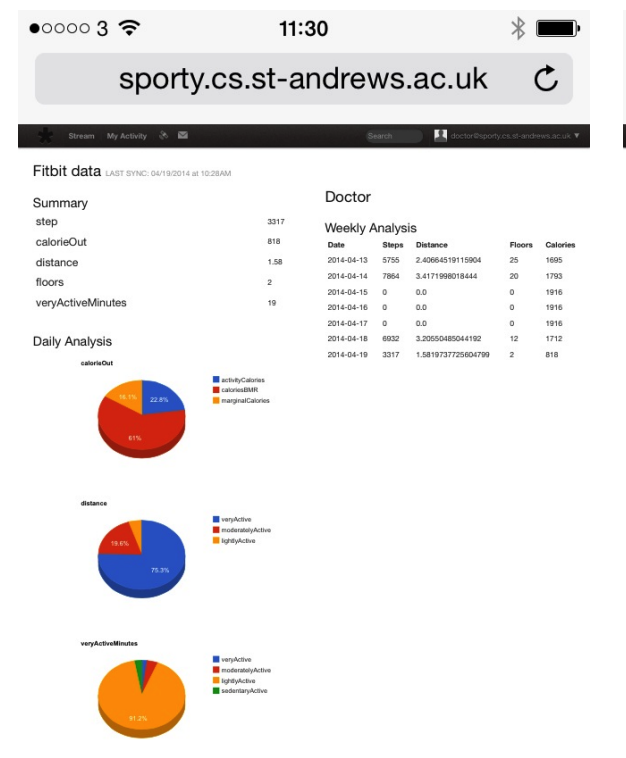

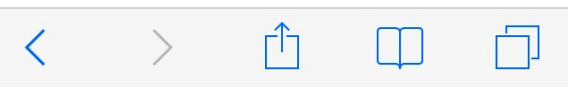

(a) Doctor viewpoint. Full detailed access to Fitbit data, including historical data.
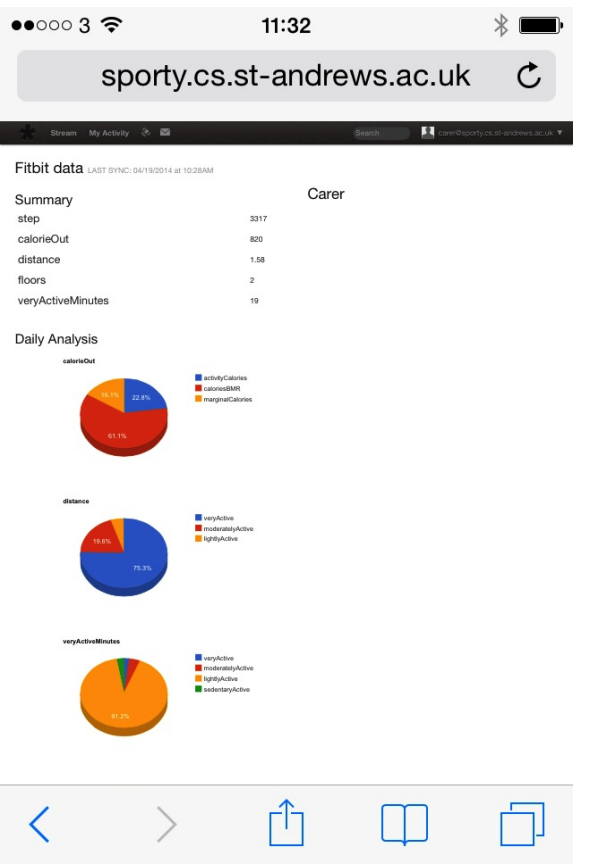

(b) Carer viewpoint. Partial detailed access to

Fitbit data, including historical data. Fitbit data, no historical but daily data. tailed access to Fitbit data, today's data only.
Fig. 3: Example Diaspora application viewpoints (doctor, carer and patient). We show a view from a mobile device, but with
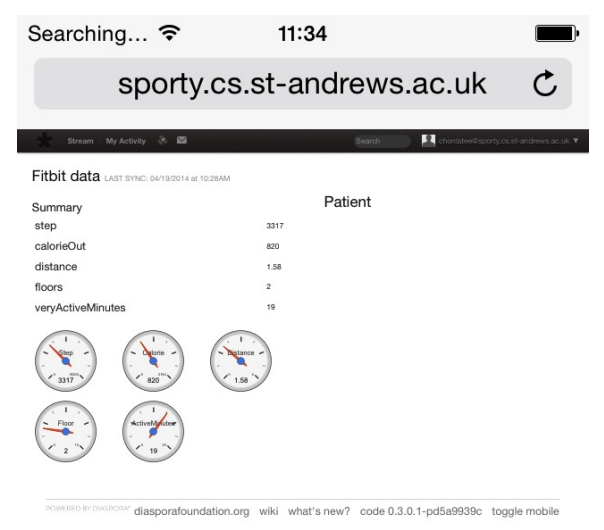
a web-based interface, non-mobile devices can also access the data. All views are configurable subject to appropriate policy: we have presented examples here to show the concept.

When ambulatory monitoring and care is used, patients are willing to share their related health data for their own safety and benefit, e.g. patients having heart disease would not mind sharing their heart-rate data for the use of heart rate monitoring for their own health. However, besides the heartrate data which is linked directly to the diagnosis of heart disease, there is also other additional health information which can help physicians to better diagnose patients health status. Therefore, it is of interest whether and to which extent people are willing to share these additional private but useful data in the trusted environment of a carer network.

In an mHealth scenario like heart rate monitoring [46], ECG data is considered as the main parameter to indicate a symptom of heart failure or arrhythmias, whereas other parameters like blood pressure, body weight, age and physical activity are considered as risk factors which are useful for physicians for better diagnosis and prevention. Similarly, in our parallel wellbeing scenario, the steps count from Fitbit is considered as main information required for the trainer and coach, as a direct indication of fitness level achieved by clients. The sleeping pattern is considered as an additional piece of information which clients need not to share since it is not related directly to their goals and achievement. However, sleeping pattern is also important and can indicate the state of being healthy. According to [47], sleeping quantity and quality can affect health outcomes. Considered as an important parameter for wellbeing monitoring, a mobile application developed in [36] also included sleep as a wellbeing indicator.

Therefore, in our wellbeing user study, the sleep pattern is included as a additional information helping diagnosis.
However, users will have options whether to share their sleep patterns, i.e. private but useful data, to buddies and/or fitness professionals (trainers and coaches).

Figure 4 shows the key interfaces we use for investigation of users sharing behaviour. We are interested in the data flow from users to two different interfaces with respect to privacy: personal to professional actors, and personal to personal actors. Our purpose is to find out the variation in clients/patients concerns in sharing of their private, useful but non-mandatory data (sleep pattern) with professionals (trainer and coach) and with personal actors (buddy) in a the environment of a carer (fitness) network.

\section{B. Comparison with Fitbit native apps}

The Fitbit device is accompanied by a a web-portal and smartphone application that allows sharing of data. However, we use it as a measurement device and instead use a front-end based on a modified Diaspora platform and our carer network. In summary, the important functionality offered by the original Fitbit application (web portal) compared with our modified Disapora OSMP is as follows.

Privacy of data sharing and level of users control. Fitbit does not allow a fine-grained level of control for sharing of data. Users have choices only to share with their friends, public or not to share at all. In our Diaspora platform, users have more control over sharing of their own data at a fine-grained level, i.e. users can select with whom they want to share as well as adjust the frequency of sharing. In accordance with a study by Prasad et al [25], to enable fine-grained level of user control is essential for the acceptance and use of mHealth application. 


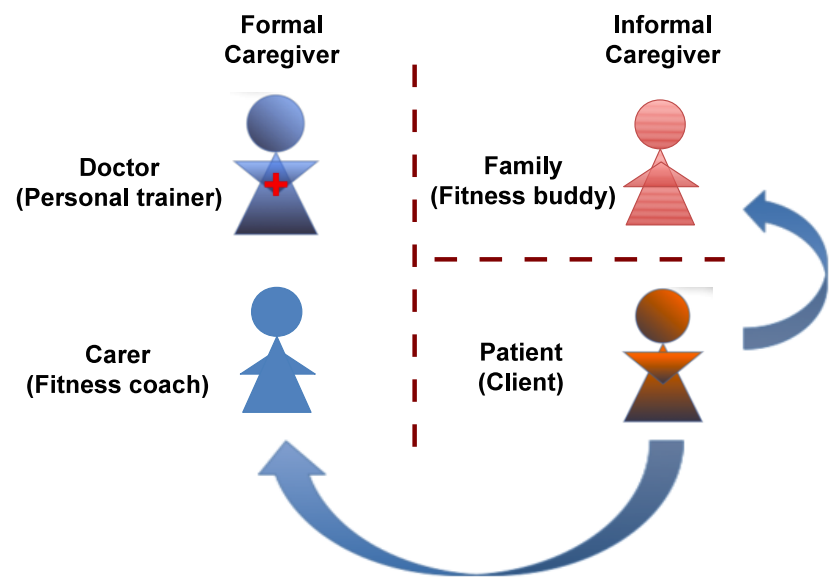

Fig. 4: Privacy and data sharing interfaces we consider in carer network. There are two key interfaces we test: 1) between professional relationships, i.e. data flow from a patient to formal caregivers like doctor and carer; 2) between personal relationships, i.e. from a patient to non-healthcare professional or informal caregiver like family member (buddy).

Motivation and community. Among available activity tracker devices and applications, Fitbit is well-known for using 'gamification' for motivation and changing people behaviours, e.g. a 'new badge' rewards and weekly progress emails, competitions and comparative performance with friends as well as sharing data to existing social networks, like facebook. In general, motivation and feedback in a native Fitbit application is based on self-setting goals, with feedback notifications and rewards automatically set by application. Friends and community are limited only to other Fitbit users. In comparison, the use of Diaspora and a carer network provides a collaborative community with a closed-loop feedback, i.e. enable better communication and collaboration between users and all other trusted actors. Users can receive feedback via both automatically generated alerts and human generated messages.

Data viewpoint. Fitbit provides a well-designed dashboard with a graphical interface, using widgets such as meters and bar charts, as well as detailed logs of activities to help users interpret and understand their data. In Fitbit the application, users have access to all their own detailed data, whereas in the Diaspora application, different actors have different levels of access based on their requirements. Such requirements are configurable, of course. We have chosen, arbitrarily, to allow actors to access only the part of the information necessary and required for their roles in a carer network. For example, patients/clients need not see their step counts and active minutes in detail, but might want to see only the summary messages shwoing their progress wiht respect to their goals. However, a trainer and coach may require more details for performance analysis. The same approach applies also to the control and management interfaces of the application, i.e. clients have only a simple turn on/off control, while a trainer has the most in-depth level of access and control over the application based on their roles of being in charge of the exercise programme.
Data export. Despite the well-provided portal for monitoring users data in a dashboard, to export data out of Fitbit is not easy. There is no web interface for users to download data, but a set of free available APIs. This requires developers to build applications to access the data with authorisation of users. Otherwise, data export is possible if users pay extra costs, i.e. a Fitbit 'premium' feature. Nevertheless, the data which can be accessed is only the daily summary of steps (also stairs climbed and calories burned). To be able to access the detailed, minute-by-minute data, a user must apply to Fitbit to access the 'partner level' API. According to the concern expressed in [48], Fitbit users have no access to their own data while companies make profits from users' data. The use of third party mobile applications for pervasive monitoring can therefore violate easily the privacy of users. For a deployment use of the Diaspora platform in a real mHealth application, the data download function is possible via the open source nature of Diaspora. Although this makes no difference in the designed experiment since data comes from the Fitbit server, in a real mHealth scenario, the use of open source and publicly accessible platform like Diaspora would enable a greater set of privacy and security controls, as well as confirming with any national freedom of information or data protection laws.

\section{CONCLUSION}

In this work, we have proposed and examined the use of wellbeing devices and applications, Fitbit, with an open source online social media platform, Diaspora, to create a proxy for an mHealth application scenario. We leverage the public interest in the quantified self to the benefit of citizens and show that it is possible to build an analogy between wellbeing and mHealth scenarios for the benefit of experimentation and research, without the overhead and complexity of clinical constraints. This enables reserachers to focus on new technology and systems aspects, without losing context, to be able to make faster progress in pre-clinical settings. Our example study is on the investigation of privacy and information sharing in a trusted environment of a remote monitoring application for mHealth. We have presented arguments that the sharing behaviour in both mHealth monitoring and purpose-oriented wellbeing monitoring is similar, i.e. if the context of data sharing is appropriate to the shared environment, then the trust relationships that exist are similar. We believe that the use of wellbeing monitoring as a proxy experiment for mHealth monitoring would make trials in the early research stages more possible and facilitate researches in this area for advancing future mHealth systems.

\section{REFERENCES}

[1] L. M. Gutiérrez-Robledo, "Looking at the future of geriatric care in developing countries," The Journals of Gerontology Series A: Biological Sciences and Medical Sciences, vol. 57, no. 3, pp. M162-M167, 2002.

[2] C. Lionis and E. Petelos, "The impact of the financial crisis on the quality of care in primary care: an issue that requires prompt attention," Quality in primary care, vol. 21, no. 5, pp. 269-273, 2013.

[3] C. Khorakhun and S. N. Bhatti, "Remote Health Monitoring Using Online Social Media Systems," in Proc. 6th IFIP/IEEE Wireless and Mobile Networking Conference (WMNC2013), Apr 2013. 
[4] _ , "Alerts for Remote Health Monitoring Using Online Social Media Platforms," in 15th IEEE Intl. Conf. on e-Health Networking Applications and Services (HealthCom), Oct 2013.

[5] _ _ "Using Online Social Media Platforms for Ubiquitous, Personal Health Monitoring," in 16th IEEE Intl. Conf. on e-Health Networking Applications and Services (HealthCom), Oct 2014.

[6] Khorakhun, C. and Bhatti, S. N., "Remote health monitoring using online social media," EAI Endorsed Trans. Ubiquitous Environments, In press.

[7] M. Swan, "Emerging patient-driven health care models: an examination of health social networks, consumer personalized medicine and quantified self-tracking," International journal of environmental research and public health, vol. 6, no. 2, pp. 492-525, 2009.

[8] FitBit, "FitBit," http://www.fitbit.com, (Jun 2014).

[9] Jawbone, "Jawbone Up," https://jawbone.com/up, (Jun 2014).

[10] Basis, "Basis," http://www.mybasis.com, (Jun 2014).

[11] Nike, "Nike+," https://secure-nikeplus.nike.com/plus/, (Jun 2014).

[12] Adidas, "adidas miCoach," http://micoach.adidas.com, (Jun 2014).

[13] Shine, "Shine," http://www.misfitwearables.com, (Jun 2014).

[14] Lark, "Lark," http://lark.com/Samsung/index2.html, (Jun 2014).

[15] Garmin, "Garmin," http://connect.garmin.com/en-US/, (Jun 2014).

[16] Philips, "Philips Telehealth Solution," http://telehealth.philips.com, (September 2013).

[17] DailyMile, "DailyMile," http://www.dailymile.com, (Jun 2014).

[18] RunKeeper, "RunKeepper," http://runkeeper.com, (Jun 2014).

[19] LoseIt, "LoseIt," http://www.loseit.com, (Jun 2014).

[20] AgaMatrix, "AgaMatrix," http://agamatrix.com/medical-devices/, (Jun 2014).

[21] M. Boulos, S. Wheeler, C. Tavares, and R. Jones, "How smartphones are changing the face of mobile and participatory healthcare: an overview, with example from eCAALYX," Biomedical engineering online, vol. 10, no. 1, p. 24, 2011.

[22] B. Silva, I. Lopes, J. Rodrigues, and P. Ray, "SapoFitness: A mobile health application for dietary evaluation," in 13th IEEE Intl. Conf. on e-Health Networking Applications and Services (HealthCom), 2011, pp. 375-380.

[23] G. Walsh and J. Golbeck, "Stepcity: a preliminary investigation of a personal informatics-based social game on behavior change," in CHI'14 Extended Abstracts on Human Factors in Computing Systems. ACM, 2014, pp. 2371-2376.

[24] P. Pendse and J. Greene, "A wellness android application with social networking capabilities," in Proceedings of the 51st ACM Southeast Conference. ACM, 2013, p. 24.

[25] A. Prasad, J. Sorber, T. Stablein, D. Anthony, and D. Kotz, "Understanding sharing preferences and behavior for mHealth devices," in Proceedings of the 2012 ACM workshop on Privacy in the electronic society. ACM, 2012, pp. 117-128.

[26] K. Tollmar, F. Bentley, and C. Viedma, "Mobile Health Mashups," in Proc. PervasiveHealth, May 2012.

[27] F. Guo, Y. Li, M. S. Kankanhalli, and M. S. Brown, "An evaluation of wearable activity monitoring devices," in Proceedings of the 1st ACM international workshop on Personal data meets distributed multimedia. ACM, 2013, pp. 31-34

[28] Jonah Comstock, "Samsung reveals Simband and Sami health platform," http://mobihealthnews.com/25126/mayo-fitbit-data-predictssurgical-recovery-time/, (Aug 2013).

[29] patientslikeme, "patientslikeme," http://www.patientslikeme.com, (Jun 2014).

[30] Doximity, "Doximity," https://www.doximity.com, (Jun 2014).

[31] Sermo, "Sermo," http://www.sermo.com, (Jun 2014).

[32] hellohealth, "hellohealth," http://hellohealth.com, (Aug 2014).

[33] M. J. Ball and J. Lillis, "E-health: transforming the physician/patient relationship," International journal of medical informatics, vol. 61, no. 1, pp. $1-10,2001$

[34] T. K. Lu, F. and B. Murphy, "Reducing Adolescent Obesity with a Mobile Fitness Application: Study Results of Youth Age 15 to 17," in 15th IEEE Intl. Conf. on e-Health Networking Applications and Services (HealthCom), Oct 2013.

[35] S. Consolvo, D. W. McDonald, T. Toscos, M. Y. Chen, J. Froehlich, B. Harrison, P. Klasnja, A. LaMarca, L. LeGrand, R. Libby et al., "Activity sensing in the wild: a field trial of ubifit garden," in Proceedings of the SIGCHI Conference on Human Factors in Computing Systems. ACM, 2008, pp. 1797-1806.
[36] N. D. Lane, M. Mohammod, M. Lin, X. Yang, H. Lu, S. Ali, A. Doryab, E. Berke, T. Choudhury, and A. Campbell, "Bewell: A smartphone application to monitor, model and promote wellbeing," in 5th International ICST Conference on Pervasive Computing Technologies for Healthcare, 2011, pp. 23-26.

[37] M. W. Newman, D. Lauterbach, S. A. Munson, P. Resnick, and M. E. Morris, "It's not that i don't have problems, i'm just not putting them on facebook: challenges and opportunities in using online social networks for health," in Proceedings of the ACM 2011 conference on Computer supported cooperative work. ACM, 2011, pp. 341-350.

[38] D. A. Epstein, A. Borning, and J. Fogarty, "Fine-grained sharing of sensed physical activity: a value sensitive approach," in Proceedings of the 2013 ACM international joint conference on Pervasive and ubiquitous computing. ACM, 2013, pp. 489-498.

[39] K. Wild, L. Boise, J. Lundell, and A. Foucek, "Unobtrusive in-home monitoring of cognitive and physical health: Reactions and perceptions of older adults," Journal of Applied Gerontology, vol. 27, no. 2, pp. 181-200, 2008

[40] U. Anliker, J. A. Ward, P. Lukowicz, G. Troster, F. Dolveck, M. Baer, F. Keita, E. B. Schenker, F. Catarsi, L. Coluccini et al., "AMON: a wearable multiparameter medical monitoring and alert system," Information Technology in Biomedicine, IEEE Transactions on, vol. 8, no. 4, pp. 415-427, 2004.

[41] M. Blount, V. M. Batra, A. N. Capella, M. R. Ebling, W. F. Jerome, S. M. Martin, M. Nidd, M. R. Niemi, and S. P. Wright, "Remote healthcare monitoring using Personal Care Connect," IBM Systems Journal, vol. 46, no. 1, pp. 95-113, 2007.

[42] A. Wood, G. Virone, T. Doan, Q. Cao, L. Selavo, Y. Wu, L. Fang, Z. He, S. Lin, and J. Stankovic, "ALARM-NET: Wireless sensor networks for assisted-living and residential monitoring," University of Virginia Computer Science Department Technical Report, vol. 2, 2006.

[43] G. R. Hayes, K. G. Cheng, S. H. Hirano, K. P. Tang, M. S. Nagel, and D. E. Baker, "Estrellita: A mobile capture and access tool for the support of preterm infants and their caregivers," ACM Transactions on Computer-Human Interaction (TOCHI), vol. 21, no. 3, p. 19, 2014.

[44] N. Carpentier and F. Ducharme, "Care-giver network transformations: the need for an integrated perspective," Ageing and Society, vol. 23, no. 04 , pp. 507-525, 2003.

[45] M. S. M. M. Y. M. Carol Kennedy-Armbruster, Ph.D. and B. Hannah Calvert, "Apersonal training and fitness coaching: Do they really work?" in Certifiednews. American Council on Ecercise (ACE), Jan 2012.

[46] F. Koehler, S. Winkler, M. Schieber, U. Sechtem, K. Stangl, M. Böhm, H. Boll, G. Baumann, M. Honold, K. Koehler et al., "Impact of remote telemedical management on mortality and hospitalizations in ambulatory patients with chronic heart failure the telemedical interventional monitoring in heart failure study," Circulation, vol. 123, no. 17, pp. 1873-1880, 2011.

[47] G. G. Alvarez and N. T. Ayas, "The impact of daily sleep duration on health: a review of the literature," Progress in cardiovascular nursing, vol. 19, no. 2, pp. 56-59, 2004.

[48] Christian Payne, "How activity trackers remove our rights to our most intimate data," http://www.theguardian.com/technology/2014/jun/03/howactivity-trackers-remove-rights-personal-data, (Jun 2014). 